phys. stat. sol. (b) 197,369 (1996)

Subject classification: 71.38 and $73.61 ;$ S7.12

Department of Physics, Bilkent University, Ankara ${ }^{1}$ )

\title{
Variational Approach for Phonon Renormalization Effects in Photoexcited Quantum Wires and Quantum Wells
}

\author{
By \\ K. GÜven and B. TANATAR \\ (Received January 16, 1996; in revised form July 11, 1996)
}

\begin{abstract}
We investigate the effects of screening on polaronic corrections to the effective band edge in photoexcited quasi-one-dimensional GaAs quantum wires and two-dimensional quantum wells. We develop a variational method to calculate the polaron energy of a two-component plasma (electrons and holes) coupled to LO-phonons. Screening effects are incorporated within a dynamical scheme. We find that the screening effects and finite well width considerably reduce the polaron energy as the plasma density increases. Many-body corrections beyond the random-phase approximation are also considered.
\end{abstract}

\section{Introduction}

A high-density electron-hole plasma may be achieved under intense laser excitation of undoped semiconducting materials. In such systems, the exchange-correlation effects, the screening of the Coulomb interaction, and various single-particle properties are affected, among which the most dramatic one is the band-gap renormalization as a function of the plasma density. This phenomenon is important to determine the emission wavelength of coherent emitters as being used in semiconductors [1]. Since a substantial carrier population may be induced by optical excitation, the renormalized band gap can change the excitation processes leading to optical nonlinearities. On the other hand, the coupling between the charge carriers and LO-phonons in these systems also influences the band-gap energy and carrier effective mass. The gap between the valence and conduction bands is renormalized by the emission and absorption of LO-phonons [2]. In this paper we investigate the density dependence of the band-gap renormalization in quasione-dimensional (Q1D) photoexcited semiconductors due to the phonon effects within a variational approach which includes dynamical screening. The phonon contribution to the self-energy lowers the conduction band and raises the valence band, resulting in a narrowing of the band gap. Recent experiments [3] made available the density dependent change in band gap in GaAs based quantum wires. Our aim is to assess to what extent the band-gap renormalization is due to phonon effects.

Much effort has been devoted to the study of Q1D semiconductor structures in recent years. In these systems, based on the confinement of the charge carriers, the motion of charged particles is quantized in two transverse directions, thus the charge carriers es-

1) Bilkent, 06533 Ankara, Turkey. 
sentially move only in the longitudinal direction. Interest stems from fundamental and applied points of view, because of new physical phenomena involved and their potential applications in high-speed optoelectronic devices. Progress in the fabrication techniques such as molecular beam epitaxy and lithographic deposition have made possible the realization of such Q1D systems [4].

The energy and the effective mass of an electron in a quantum wire including the subband effects were calculated in the presence of electron-LO-phonon interaction by Degani and Hipólito [5]. The ground-state energy of the Q1D polaron gas in a rectangular quantum well wire has been calculated by Campos et al. [6], and very recently by Hai et al. [7]. The latter group has investigated the polaron energy in different quantum well wire models and the effects of screening. Coupling to phonons of a Q1D electron-hole plasma at finite temperature was considered by Güven and Tanatar [8] within the perturbation theory.

In this paper our primary aim is to develop a variational formulation of the contribution to the ground-state energy of an interacting electron-hole-phonon system. Our method is the generalization to a two-component plasma of the variational calculation of polaron energy given by Lemmens et al. [9]. Secondly, we compare our results with perturbation theory calculations to assess the validity of the static approximation to screening effects. For electron (hole)-optical phonon interaction, wave vectors such that $k \sim\left(2 m_{\mathrm{e}, \mathrm{h}} \omega_{\mathrm{LO}}\right)^{1 / 2}$ are important. The static screening approach would be valid if $k \ll k_{\mathrm{F}}$, where $k_{\mathrm{F}}$ is the Fermi wave vector. In low-dimensional (2D and Q1D) structures the plasmon energy $\omega_{\text {pl }}(k)$ is generally much smaller than the LO-phonon energy $\omega_{\mathrm{LO}}$, in contrast to the situation in the bulk. The dynamic dielectric function $\varepsilon(q, \omega)$ at finite $\omega \sim \omega_{\text {LO }}$ may differ considerably from its $\omega=0$ value (i.e., static value). Thus, the dynamical screening effects are expected to be important in low-dimensional systems as pointed out by Lei [10]. We employ a variational approach to estimate the phonon contribution to the ground-state energy, and investigate the effects of dynamic screening in various approximations. The Q1D and 2D systems studied contain electrons and holes at equal number density $N$, appropriate for an undoped, photoexcited semiconductor, which are free to move in one or two spatial directions, respectively. We consider here the coupling of electrons and holes with bulk LO-phonon modes.

\section{Theory}

The specific model we use in our calculation for the Q1D, electron-hole fluid is developed by Das Sarma and Lai [11] and is applicable to the experimental realization of semiconducting systems [12]. The charge carriers are assumed to be in a zero thickness $x y$-plane with a harmonic (parabolic) confinement potential in the $y$-direction so that the subband energies are $\varepsilon_{n}=\Omega(n+1 / 2)$, where $\Omega$ describes the strength of the confining potential. In this work, we shall assume that both types of carriers are in their lowest subbands. This approximation will hold as long as the subband separation remains much larger than the phonon energy in quantum wires and the thermal energy $k_{\mathrm{B}} T$. The Coulomb interaction between the particles in our model Q1D system is given by [13] $\left(2 e^{2} / \varepsilon_{0}\right) F(q)$, where $F(q)=\frac{1}{2} \exp \left(b^{2} q^{2} / 4\right) \mathrm{K}_{0}\left(b^{2} q^{2} / 4\right)$ in which $\mathrm{K}_{0}(x)$ is the modified Bessel function and $\varepsilon_{0}$ the background dielectric constant. The characteristic length $b=1 \sqrt{\mu \Omega}$, where $\mu$ is the reduced mass of the electron-hole pair, is related to the confining potential strengths of electrons and holes, and for simplicity we use throughout this paper the same value of $b$ for both species. For more realistic calculations this restriction may easily be relaxed. 
We study the Q1D polaron gas using the Lee-Low-Pines unitary transformation approach as introduced by Lemmens et al. [9] and $\mathrm{Wu}$ et al. [14] in application to 3D and Q2D systems. It is harder to incorporate the screening effects (especially the dynamic screening) in the perturbation theory approach $[2,8]$, thus a variational method seems more suitable. We introduce a unitary transformation, $U=\exp [Q]$, where $Q=\sum_{i, k, q} f_{q, i}\left(a_{q}-a_{-q}^{\dagger}\right) c_{k, i}^{\dagger} c_{k+q, i}$ applied to the Hamiltonian of the interacting many electron-hole and phonon system. Here the index $i$ runs over electron and hole components, and the creation and annihilation operators for different species have their usual meaning. The variational parameters $f_{q, i}$ are to be determined by minimizing the ground-state energy. Following the usual procedure $[7,9,14]$ the ground state may be written as the product of phonon vacuum state and ground-state wave function of electrons and holes, and minimizing the energy with respect to the variational parameters, we arrive at the ground-state energy of the two-component polaron gas. Minimization yields the following set of equations for the unknown variational parameters (we suppress the $q$-dependence of $M_{i}$ and $f_{i}$ ):

$$
\begin{aligned}
& \left(S_{11} \omega_{\mathrm{LO}}+\frac{q^{2}}{2 m_{1}}\right) f_{1}+S_{12} \omega_{\mathrm{LO}} f_{2}=S_{11} M_{1}+S_{12} M_{2} \\
& \left(S_{22} \omega_{\mathrm{LO}}+\frac{q^{2}}{2 m_{2}}\right) f_{2}+S_{12} \omega_{\mathrm{LO}} f_{1}=S_{22} M_{2}+S_{12} M_{1}
\end{aligned}
$$

where $S_{i j}(q)$ are the static structure factors to be discussed below. In the extreme quantum limit, when the electrons and holes are in the lowest subband, the Q1D electron (hole)-phonon interaction matrix element is given by $[2,6,7] \quad\left|M_{q, i}\right|^{2}$ $=\left[2 \alpha_{i} \omega_{\mathrm{LO}}^{2} / \sqrt{2 m_{i} \omega_{\mathrm{LO}}}\right] F(q)$. Solving the above set of coupled equations for $f_{i}$, we obtain the polaronic contribution to the ground-state energy as

$$
\begin{aligned}
E_{\mathrm{p}}= & -\sum_{q}\left\{S_{11}\left(M_{1}^{*} f_{1}+M_{1} f_{1}^{*}\right)+S_{12}\left(M_{1}^{*} f_{2}+M_{1} f_{2}^{*}+M_{2}^{*} f_{1}+M_{2} f_{1}^{*}\right)\right. \\
& \left.+S_{22}\left(M_{2}^{*} f_{2}+M_{2} f_{2}^{*}\right)\right\}+\sum_{q} \omega_{\mathrm{LO}}\left\{S_{11} f_{1}^{*} f_{1}+S_{12}\left(f_{1}^{*} f_{2}+f_{1} f_{2}^{*}\right)+S_{22} f_{2}^{*} f_{2}\right\} \\
& +\sum_{q}\left(\frac{q^{2}}{2 m_{1}} f_{1}^{*} f_{1}+\frac{q^{2}}{2 m_{2}} f_{2}^{*} f_{2}\right) .
\end{aligned}
$$

When the correlations between the electrons and holes are neglected, i.e., $S_{12}=0$, we obtain a simplified expression for the energy,

$$
E_{\mathrm{p}}=-\left\{\sum_{\mathrm{q}}\left|M_{1}\right|^{2} \frac{S_{11}^{2}}{\omega_{\mathrm{LO}} S_{11}+q^{2} / 2 m_{1}}+\sum_{q}\left|M_{2}\right|^{2} \frac{S_{22}^{2}}{\omega_{\mathrm{LO}} S_{22}+q^{2} / 2 m_{2}}\right\}
$$

as a sum of individual contributions of the plasma components. Furthermore, setting $S_{11}$ $=S_{22}=1$, amounts to the no-screening limit, and we recover the perturbation theory result.

We consider two approximations in the evaluation of static structure factors $S_{i j}(q)$. In the first case, we use the Hartree-Fock (HF) approximation, which has the simple form

$$
S_{i j}^{\mathrm{HF}}(q)=\delta_{i j} \begin{cases}q / 2 k_{\mathrm{F}} ; & q<2 k_{\mathrm{F}} \\ 1 ; & q \geq 2 k_{\mathrm{F}} .\end{cases}
$$




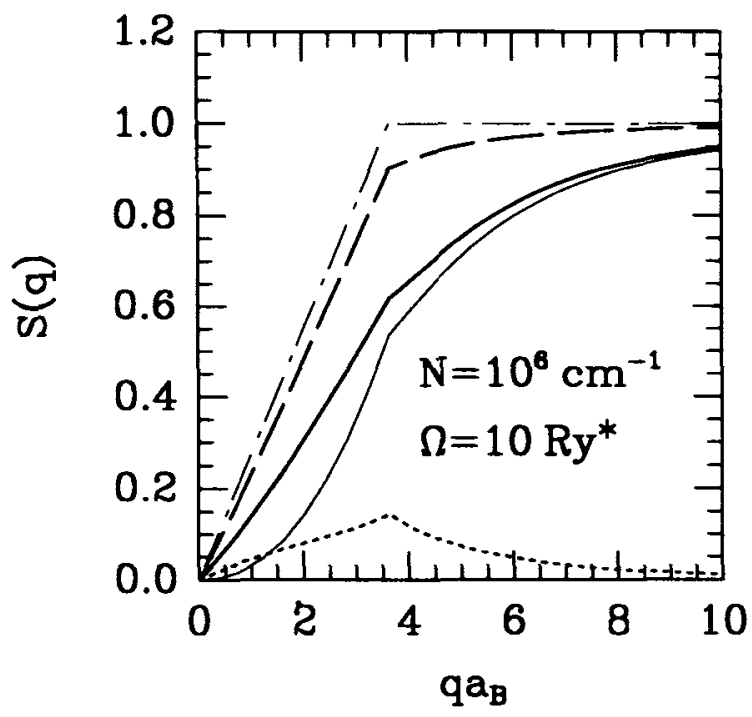

Fig. 1. Static structure factors within RPA in a Q1D electron-hole system. Solid, dashed, and dotted lines indicate $S_{11}(q), S_{22}(q)$, and $S_{12}(q)$, respectively. The dash-dotted line is for the Hartree-Fock approximation. Thin solid line is the function $D(q)=S_{11} S_{22}-S_{12}^{2}$

Note that in the HF approximation $S_{11}=S_{22}$, since we have equal numbers of electrons and holes, and $S_{12}=0$. In the second case, we employ the RPA generalized to a twocomponent system [15]. The density-density response function of the system is expressed in matrix form,

$$
\left[\chi^{\mathrm{RPA}}(q, \omega)\right]^{-1}=\left(\begin{array}{cc}
\left.\chi_{11}^{0}(q, \omega)\right]^{-1}-V(q) & -V(q) \\
-V(q) & {\left[\chi_{22}^{0}(q, \omega)\right]^{-1}-V(q)}
\end{array}\right)
$$

where $\chi_{i i}^{0}(q, \omega)$ is the Lindhard function for the $i$-th component, i.e., the noninteracting susceptibility. We calculate the corresponding static structure factors using

$$
S_{i j}^{\mathrm{RPA}}(q)=-\frac{1}{N} \int_{0}^{\infty} \frac{\mathrm{d} \omega}{\pi} \chi_{i j}^{\mathrm{RPA}}(q, \omega)
$$

where the analytic continuation of the response function to the complex frequency plane and a subsequent Wick rotation of the frequency integral are used to incorporate the single-particle and plasmon contributions.

The static structure factors $S_{i j}(q)$ are obtained from the full frequency dependent response function $\chi(q, \omega)$ by integrating over all frequencies, thus they inherently carry dynamic information. For Q1D electron systems the collective excitations (plasmons) have a strong wave vector dependence without damping. Thus, along with the singleparticle excitations, plasmons must also be taken into account in the calculation of $S_{i j}(q)$. The static structure factors, as set out above, determine the screening properties of the electron (hole)-phonon system. In Fig. 1 we show the resulting partial structure factors in a two-component plasma for a typical density $N=10^{6} \mathrm{~cm}^{-1}$ and confinement energy $\Omega=10 \mathrm{Ry}^{*}$ (the effective Rydberg is defined as $\mathrm{Ry}^{*}=e^{2} / 2 a_{\mathrm{B}}^{*}$ ). Solid, dashed, and dotted lines indicate $S_{11}(q), S_{22}(q)$, and $S_{12}(q)$, respectively, whereas the dash-dotted line is the HF result. Also shown by the thin solid line is the quantity 
$D(q)=S_{11}(q) S_{22}(q)-S_{12}^{2}(q)$ as defined by Chakraborty [16]. It has been argued that $D(q)$ qualitatively resembles the static structure factor of a single-species system of the same density.

\section{Results for Quantum Wire Structures}

We illustrate our calculations of the electron (hole)-phonon contribution to the groundstate energy of a quantum wire by choosing the GaAs system. The relevant parameters used in the calculations are $m_{1}=0.067 m_{\mathrm{e}}, m_{2}=0.5 m_{\mathrm{e}}$, for the electron and hole effective masses, respectively, $\alpha_{1}=0.07, \alpha_{2}=0.195$, for the electron-phonon and hole-phonon coupling constants, respectively, and $\omega_{\mathrm{LO}}=36.5 \mathrm{meV}$. For the above material parameters and confining potential strength $\Omega=10 \mathrm{Ry}^{*}$, the carriers remain in the lowest $1 \mathrm{D}$ subband for a wire size of roughly $b \approx 50 \AA$. We estimate the Fermi energy of the electron-hole system to be $E_{\mathrm{F}}<5 \mathrm{Ry}^{*}$ as long as the carrier density is $N<(3$ to 4) $\times 10^{6} \mathrm{~cm}^{-1}$. The single-subband approximation would break down for higher densities (i.e., $N \approx 10^{7} \mathrm{~cm}^{-1}$ ), in which case the present theory would be inapplicable.

We show in Fig. 2 the total polaronic contribution to the ground-state energy as a function of the one-dimensional electron-hole plasma density $N$. The solid line represents the variational calculation employing the RPA structure factors to account for the screening effects. The variational calculation using the Hartree-Fock structure factors is indicated by the dashed line. For comparison we also show by the dotted line the result of a perturbative calculation. We first note that both the variational RPA and the perturbative calculations exhibit considerable screening even at densities as low as $N \approx 10^{5} \mathrm{~cm}^{-1}$. For the present choice of the confining potential energy $\left(\Omega=10 \mathrm{Ry}^{*}\right)$ the unscreened polaron energy is about $-7 \mathrm{meV}$. The Hartree-Fock approximation gives relatively small screening at low density, and in general it underestimates the screening effect. The perturbative calculation we employ [8] includes the static dielectric function $\varepsilon(q, 0)$ through the renormalization of the electron (hole)-phonon interaction matrix element $\left|M_{1}\right|^{2} /[\varepsilon(q, 0)]^{2}$. We use the $T=0$, plasmon-pole approximation for $\varepsilon(q, \omega=0)$

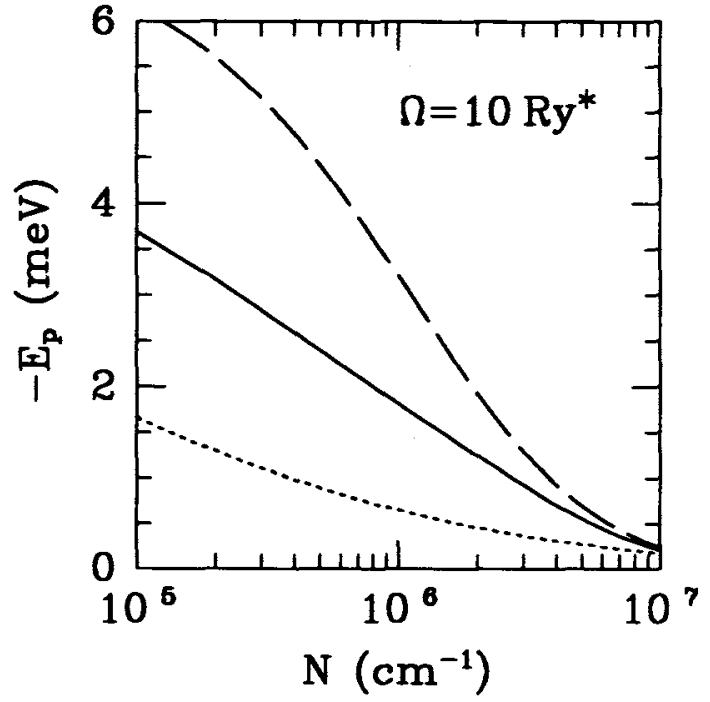

Fig. 2. Polaronic contribution to the ground-state energy for $\Omega=10 \mathrm{Ry}^{*}$ as a function of plasma density $N$. The solid and dashed lines indicate the variational calculation using the RPA and HartreeFock structure factors, respectively. The dotted line is for the perturbative calculation using the plasmon-pole approximation 


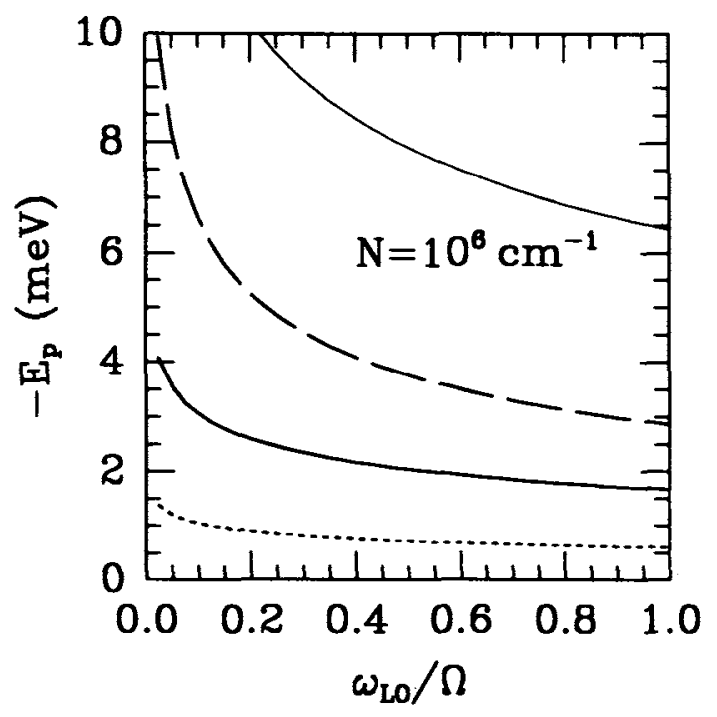

Fig. 3. Polaronic contribution to the ground-state energy for $N=10^{6} \mathrm{~cm}^{-1}$ as a function of $\omega_{\mathrm{LO}} / \Omega$. The solid and dashed lines indicate the variational calculation using the RPA and Hartree-Fock structure factors, respectively. The dotted line is for the perturbative calculation using the plasmon-pole approximation. The thin solid line represents the unscreened limit

which includes the contribution of electrons and holes,

$$
\varepsilon(q, 0)=1+\sum_{i=e, \mathrm{~h}} \frac{\left[\omega_{\mathrm{pl}}^{i}(q)\right]^{2}}{\left(q^{2} / 2 m_{i}\right)^{2}},
$$

where the Q1D plasmon frequency is $\left(\omega_{\mathrm{pl}}^{i}(q)\right)^{2}=N\left(q^{2} / 2 m_{i}\right) V(q)$. As in the case of quantum wells [2] (2D structures), the static approximation overestimates the effects since only the long-time response of the system is accounted for within this approach. We observe that going from the HF to RPA, the screening reduces the electron (hole)phonon interaction appreciably for low carrier densities. It has been noted [2] that the static screening has a stronger effect on the renormalization (of polaron energy and mass) than the dynamic screening, because in the static approximation only the longtime response of the system is taken into account. Similar conclusions are drawn by Hai et al. [7] in a calculation that takes the dynamic screening effects into account for singlecomponent Q1D systems.

In Fig. 3, the confining potential energy dependence (or size dependence) of the polaronic contribution to the ground-state energy is illustrated. We show the results of various approximations as a function of $\omega_{\mathrm{LO}} / \Omega$ at a fixed plasma density, $N=10^{6} \mathrm{~cm}^{-1}$. The solid and dotted lines represent the dynamical (variational, RPA), and static (perturbative, plasmon-pole approximation) screening calculations, respectively. We again observe that static approximation overestimates screening effects in comparison to the dynamical approach. The variational calculation using the HartreeFock approximation to the structure factors (dashed line) appears to underestimate the screening effects especially for wide quantum wires (small $\Omega$ ). The thin solid line indicates the unscreened polaron energy.

For the Q1D electron system we have used a parabolic confinement potential model. There are various other models of the quantum well wire structures making use of infinite barrier potentials and geometrical reduction of dimensionality [7]. The general trends obtained here for the carrier density and screening dependence should be valid irrespective of the details of the model chosen. We have used the RPA to describe 


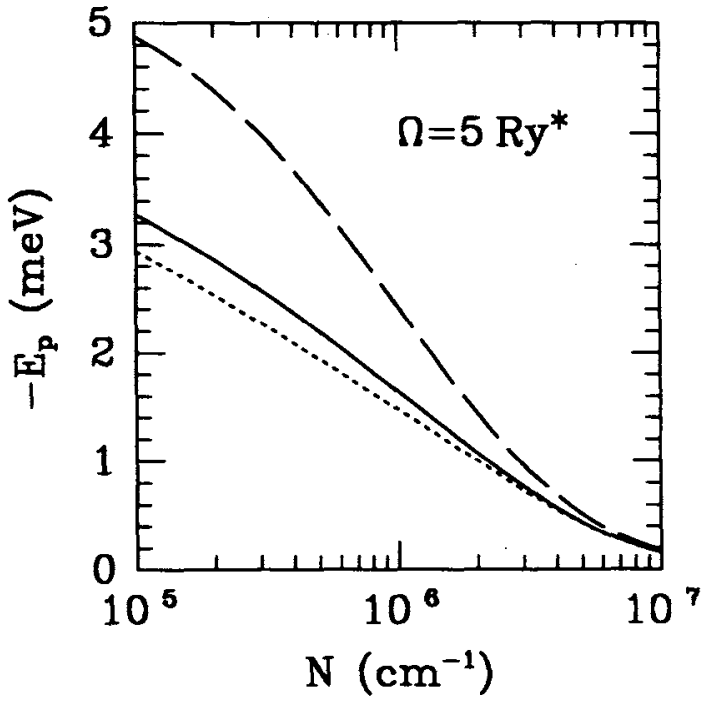

Fig. 4. Effect of local field correction on the polaron energy. The solid and dotted lines are calculated with and without $G(q)$, respectively, whereas the dashed line is the result of Hartree-Fock approximation

many-body effects in the interacting system of electrons and holes rather uncritically. It may be argued that the attractive nature of the electron-hole interaction would make the two-component plasma structure factor calculations somewhat less reliable. It is known that the corrections to RPA become more important in lower dimensions than in 3D. Also, the RPA, although exact in the high density limit, fails to take the shortrange electron correlations into account properly in the lower density regime. For these reasons it would be worthwhile to investigate corrections to RPA through local-field factors using for instance the self-consistent field method of Singwi et al. [17]. The ground-state properties including the exchange-correlation effects in quantum well wires beyond the RPA were recently studied by Campos et al. [18]. To assess the importance of local field corrections, we use the equivalent of Hubbard approximation in one dimension given as

$$
G_{i j}^{\mathrm{H}}(q) \approx \frac{1}{2} \frac{v_{i j}\left(\sqrt{q^{2}+k_{\mathrm{F}}^{2}}\right)}{v_{i j}(q)} \delta_{i j},
$$

which takes only the exchange effects into account, neglecting the Coulomb correlations. Fig. 4 shows $E_{\mathrm{p}}$ as a function of plasma density for a quantum wire with $\Omega=5 \mathrm{Ry}^{*}$. The dashed line is calculated using the HF structure factors. The solid and dotted lines are with and without the local-field factor $G(q)$, respectively. We note that the local field effects start to become important for densities less than $\approx 10^{6} \mathrm{~cm}^{-1}$. We had found that [8] vertex corrections introduced within the perturbation theory did not affect $E_{\mathrm{p}}$ appreciably.

We point out that with the present method (variational) the polaronic energy in an electron-hole system is calculated at $T=0$. It is possible to use a temperature dependent dielectric function $\varepsilon(q, T)$ within the perturbation theory approach $[2,8]$. For the variational calculation, not only the temperature dependent structure factors are necessary, but also the assumptions about the product form of the ground state need to be justified. We have neglected the interface phonon modes, which are expected to be appreciable only for very narrow wires since they have exponentially decaying amplitudes. 


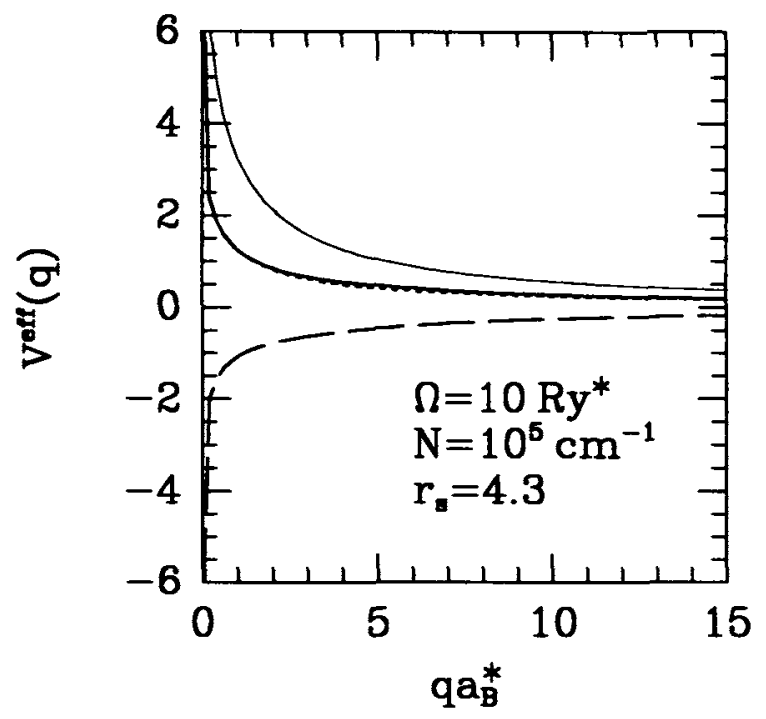

Fig. 5. The effective potential between the charge carriers at $N=10^{5} \mathrm{~cm}^{-1}$ and $\Omega=10 \mathrm{Ry}^{*}$. Solid and dotted lines are for $V_{11}^{\text {eff }}$ and $V_{22}^{\text {eff }}$, and the dashed line is for $V_{12}^{\text {eff }}$. The thin solid line is the bare Coulomb interaction

Our variational approach yields also effective interactions among the charge carriers modified by the interaction with phonons. In terms of the variational parameters $f_{i}$ they are given by

$$
V_{i j}^{\mathrm{eff}}(q)=v_{i j}(q)-2\left[M_{i}^{*} f_{j}+M_{i} f_{j}^{*}-\omega_{\mathrm{LO}} f_{i} f_{j}^{*}\right]
$$

where $v_{i j}(q)$ is the bare Coulomb interaction between different species. This result is the generalization of the effective potential as derived by Lemmens et al. [9] and da Costa and Studart [19]. We display in Fig. 5 the effective interactions for $N=10^{5} \mathrm{~cm}^{-1}$ and $\Omega=10 \mathrm{Ry}^{*}$ within the RPA. The solid and dotted lines are for $V_{11}^{\text {eff }}$ and $V_{22}^{\text {eff }}$, and the dashed line is for $V_{12}^{\text {eff }}$. We have also shown, by the thin solid line, the bare Coulomb interaction for comparison. We find that the changes due to electron (hole)-phonon interactions are significant, but decrease with increasing width (small $\Omega$ ) and increasing plasma density.

\section{Comparison with Two-Dimensional Quantum Wells}

The effects of screening on polaronic corrections to the effective band edges in Q2D quantum wells were considered by Das Sarma and Stopa [2]. They use the perturbative approach of evaluating the leading-order self-energy including the static dielectric function, and a variational formalism involving the structure factor. Their approximation amounts to the $2 \mathrm{D}$ version of our simplified expression given in (3). It is of interest to apply the full dynamical screening effects within the variational approach to quantum well structures. Fig. 6 shows the results of our dynamically screened calculation for a strictly $2 \mathrm{D}$ system, for which the Coulomb interaction is taken to be $v(q)=2 \pi e^{2} / q$. The solid and dashed lines indicate the RPA and HF, respectively, for the total (electron and hole) polaronic correction to the ground-state energy. We observe features qualitatively similar to the Q1D case, in that the RPA yields more screening than the HF approximation. The dotted line appearing in Fig. 6 is for the statically screened perturbative calculation. At zero temperature, the static dielectric function for a $2 \mathrm{D}$ system is independent 


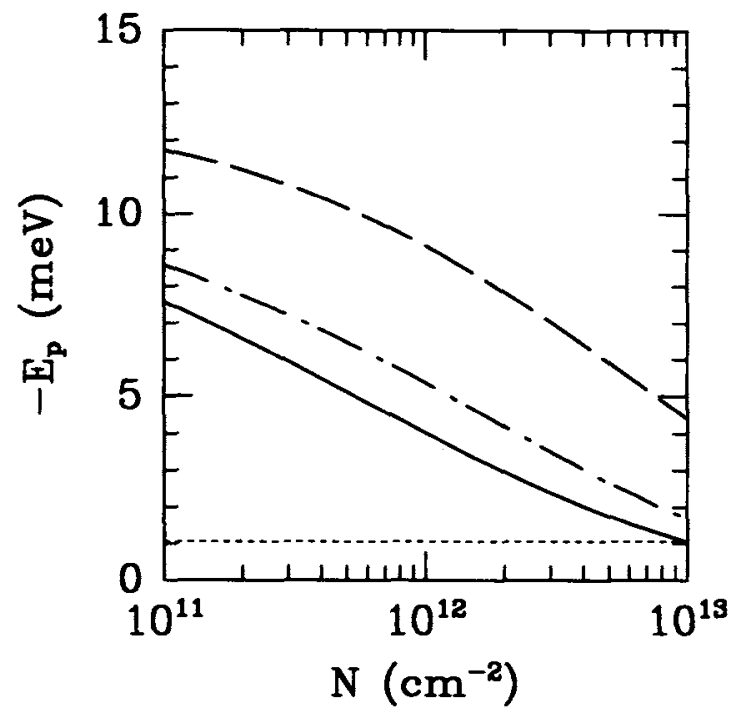

Fig. 6. Polaronic contribution to the ground-state energy in a strictly $2 \mathrm{D}$ system, as a function of plasma density. Solid and dashed lines are calculated using the RPA and Hartree-Fock structure factors, respectively, within the variational approach. The dotted line is the result of the perturbative calculation including static screening. The dash-dotted line shows the variational approach in RPA without the cross term $S_{12}(q)$

of density. Note that the $2 \mathrm{D}$ screening wave vector is given by $q_{\mathrm{s}}=2 m e^{2} / \varepsilon_{0}$. Thus, we have a constant line which nevertheless exhibits considerable screening (over screening) as noted by Das Sarma and Stopa [2]. Deviations from the constant behavior could be attributed to finite temperature effects. The dash-dotted line in Fig. 6 is evaluated with the aid of (3) when the correlations between electrons and holes are neglected. We find that omitting $S_{12}(q)$ in (2) affects the polaronic contribution to the energy considerably. We next study the effects of finite well width on the energy $E_{\mathrm{p}}$. Assuming that only the lowest subband is occupied both in conduction and valence bands, we use the form factor $[2,20]$

$$
F(q)=\frac{8}{q^{2} a^{2}+4 \pi^{2}}\left[\frac{3}{8} q a+\frac{\pi^{2}}{q a}-\frac{4 \pi^{2}\left(1-\mathrm{e}^{-q a}\right)}{q^{2} a^{2}\left(q^{2} a^{2}+4 \pi^{2}\right)}\right],
$$

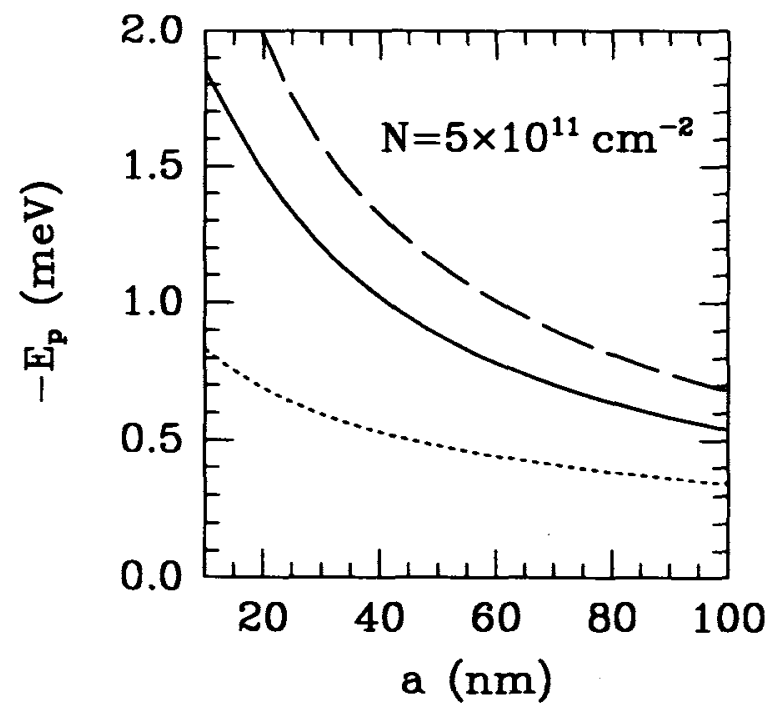

Fig. 7. The size dependence of the polaronic contribution in a quantum well structure. Solid and dashed lines are calculated using RPA and Hartree-Fock structure factors, respectively. The result of the perturbative calculation is indicated by the dotted line 
where $a$ is the well width. In Fig. 7 we display the polaronic contribution to the groundstate energy as a function of quantum well width at a typical plasma density $N=5 \times 10^{11} \mathrm{~cm}^{-2}$. We note that the screening effects dominate as the well width is increased. Similar conclusions may be drawn from the calculations of Das Sarma and Stopa [2]. A more complete many-body calculation within the perturbation theory of the band-gap renormalization which includes the electron-electron and electron-phonon interactions for semiconductor quantum wells was performed by Das Sarma et al. [21].

\section{Conclusion}

In summary, we have calculated the polaron energy of an electron-hole system coupled to bulk LO-phonons in a Q1D GaAs quantum well wire at zero temperature, within a dynamical scheme taking into account the full frequency dependence of the dielectric response. The variational method of Lemmens et al. [9] has been extended to the case of a two-component plasma interacting with phonons. The screening effects due to interactions between electrons and holes in a photoexcited quantum wire are incorporated into the electron (hole)-phonon interaction within the HF and RPA. Our results show that the dynamical screening of the two-component plasma reduces the electron (hole)-phonon interaction considerably both for quantum wires and quantum wells. As the plasma density increases, viz., $N \rightarrow \infty$, the polaronic corrections to the ground-state energy vanish. For very high densities the single-subband approximation will already break down, hence our theory is restricted to $N<4 \times 10^{7} \mathrm{~cm}^{-1}$. Corrections to the RPA, introduced via simple local field factors, do not affect the polaron energy significantly.

Acknowledgements This work is supported in part by the Scientific and Technical Research Council of Turkey (TUBITAK) under Grant No. TBAG-AY/77. We are indebted to Prof. N. Studart for his suggestions. We acknowledge interesting discussions with Prof. G. D. Mahan, A. Erçelebi, and T. Hakioğlu.

\section{References}

[1] H. HAUG and S. SchmiTT-RINK, Progr. Quantum Electronics 9, 3 (1984).

S. Schmttt-Rink, D. S. Chemla, and D. A. B. Miller, Adv. Phys. 38, 89 (1989).

[2] S. Das Sarma and M. Stopa, Phys. Rev. B 36, 9595 (1987).

[3] R. Cingolani, R. Rinaldi, M. Ferrara, G. C. la Rocca, H. lage, D. Heitmann, K. Ploog, and H. Kalt, Phys. Rev. B 48, 14331 (1993).

R. Cingolani, H. Lage, L. Tapfer, H. Kalt, D. Heitmann, and K. Ploog, Phys. Rev. Letters 67, 891 (1991).

[4] A. Schmeller, A. R. Gõni, A. Pinczuk, J. S. Whiner, J. M. Calleja, B. S. Dennis, L. N. Pfeiffer, and K. W. West, Phys. Rev. B 49, 14778 (1994).

[5] M. H. Degani and O. Hipólito, Solid State Commun. 65, 1185 (1988).

[6] V. B. Campos, M. H. Degani, and O. Hipólito, Solid State Commun. 79, 473 (1991).

[7] G. Q. Hai, F. M. Peeters, J. T. Devreese, and L. Wendler, Phys. Rev. B 48, 12016 (1993).

[8] K. Güven and B. Tanatar, Phys. Rev. B 51, 1784 (1995).

[9] L. F. Lemmens, J. T. Devreese, and F. Brosens, phys. stat. sol. (b) 82, 439 (1977).

[10] X. L. LeI, J. Phys. C 18, L731 (1985).

[11] S. Das Sarma and W. Y. LaI, Phys. Rev. B 32, 1401 (1985).

[12] T. Demel, D. Heitmann, P. Grambow, and K. Ploog, Phys. Rev. B 38, 12732 (1988).

[13] G. Y. Hu and R. F. O'Connell, Phys. Rev. B 42, 1290 (1990). 
[14] X. G. Wu, F. M. Peeters, and J. T. Devreese, phys. stat. sol. (b) 133, 229 (1986).

15] P. Vashishta, P. Bhattacharyya, and K. S. Singwi, Phys. Rev. B 10, 5108 (1974).

[16] T. Chakraborty, Phys. Rev. B 26, 6131 (1982); 29, 3975 (1984).

[17] K. S. Singwi, M. P. Tosi, R. H. LAND, and A. SJölander, Phys. Rev. 176, 589 (1968).

[18] V. B. Campos, M. H. Degani, and O. Hipólito, Superlattices and Microstructures 17, 85 (1995).

A. N. Borges, M. H. Degani, and O. Hipólito, Superlattices and Microstructures 13, 375 (1993).

[19] W. B. da Costa and N. Studart, Phys. Rev. B 47, 6356 (1993).

[20] S. Das Sarma and B. A. Mason, Ann. Phys. 163, 78 (1985).

[21] S. Das Sarma, R. Jalabert, and S.-R. ERIC Yang, Phys. Rev. B 39, 5516 (1989); 41, 8288 (1990). 